Article

\title{
Spacetime Foam: From Entropy and Holography to Infinite Statistics and Nonlocality
}

\section{Y. Jack Ng}

Institute of Field Physics, Department of Physics and Astronomy, University of North Carolina, Chapel Hill, NC 27599, USA

E-mail: yjng@physics.unc.edu

Received: 25 March 2008; in revised form: 9 October 2008 / Accepted: 10 October 2008 / Published: 14 October 2008

\begin{abstract}
Due to quantum fluctuations, spacetime is foamy on small scales. The degree of foaminess is found to be consistent with holography, a principle prefigured in the physics of black hole entropy. It has bearing on the ultimate accuracies of clocks and measurements and the physics of quantum computation. Consistent with existing archived data on active galactic nuclei from the Hubble Space Telescope, the application of the holographic spacetime foam model to cosmology requires the existence of dark energy which, we argue, is composed of an enormous number of inert "particles" of extremely long wavelength. We suggest that these "particles" obey infinite statistics in which all representations of the particle permutation group can occur, and that the nonlocality present in systems obeying infinite statistics may be related to the nonlocality present in holographic theories. We also propose to detect spacetime foam by looking for halos in the images of distant quasars, and argue that it does not modify the GZK cutoff in the ultra-high energy cosmic ray spectrum and its contributions to time-offlight differences of high energy gamma rays from distant GRB are too small to be detectable.
\end{abstract}

Keywords: spacetime foam, quantum foam, holography, dark energy, infinite statistics, nonlocality

There is no excellent beauty that hath not some strangeness in the proportion. 


\section{Introduction}

Following John Wheeler, many physicists (including the author) believe that space is composed of an ever-changing arrangement of bubbles called spacetime foam, also known as quantum foam. To understand the terminology, let us consider the following simplified analogy which Wheeler gave in a conference on gravity at University of North Carolina in 1957. Imagine yourself flying an airplane over an ocean. At high altitude the ocean appears smooth. But as you descend, it begins to show roughness. Close enough to the ocean surface, you see bubbles and foam. Analogously, spacetime appears smooth on a large scale, but on sufficiently small scales, it will appear rough and foamy, hence the term "spacetime foam" [1]. Many physicists believe the foaminess is due to quantum fluctuations of spacetime, hence the alternative term "quantum foam." This reveiw article is devoted to a discussion of spacetime foam models, or rather, a specific one of them for which the concept of entropy plays a crucial role [2-6]. The only ingredients we use in the whole discussion are quantum mechanics and general relativity, the two pillars of modern physics. We hope that the results are very general and have wider validity than most of the candidates of quantum gravity theory. Assuming that there is a unity of physics connecting the Planck scale to the cosmic scale, we also apply quantum foam physics to cosmology [7-9].

In the next section, by a process of mapping the geometry of a region of spacetime, we show how foamy spacetime is. It turns out that the degree of foaminess of spacetime determines the maximum amount of entropy a spatial region can hold. This maximum amount of information can be shown to be consistent with that encoded in the holographic principle which has its origin in black hole physics. Appropriately this spacetime foam model has come to be known as the holographic model. In section 3 , we propose to probe spacetime foam by looking for halos in the images of distant quasars or bright active galactic nuclei (AGN). There we show that the archive from the Hubble Space Telescople (HST) can already be used to yield useful bounds. Among the spacetime foam models, the holographic foam model is unique in its correspondence with the case of maximum energy density that a spatial region can hold without collapsing into a black hole. Applied to cosmology it "predicts" a critical energy density as observed in recent years. This potential connection between the extremely large and the extremely small is explored in section 4; there we show that the archive on quasars and AGN from HST can be used, in conjuction with the physics of quantum computation, to "prove" the existence of dark energy, independent of the various cosmological observations of recent years. Indeed, from our perspective, dark energy is arguably a cosmological manifestation of quantum foam! In section 5, we examine how and why dark energy is so different from ordinary energy/matter. There we show that the holographic model of spacetime foam naturally leads us to speculate that dark energy consists of an incredibly large number of extremely-long-wavelength "particles". Then we exploit the positivity of the entropy for these particles to show that they obey not the ordinary bose or fermi statistics, but the exotic infinite statistics (also known as quantum Boltzmann statistics). We start our discussion with holography and end with infinite statistics. We conclude section 5 by speculating that the nonlocality known to be present in both of them may be related to each other. We give a summary in section 6 .

For completeness, we discuss various other relevant topics of spacetime foam physics in six short appendices. In Appendix A, using a gedanken experiment to measure distances we rederive the holo- 
graphic foam model. In Appendix B, we apply the results in Appendix A to set bounds on the accuracies of clocks and limits on computations, and derive the black hole entropy and lifetime. We derive the holographic principle and the Margolus-Levitin theorem (which is liberally used in the text) in Appendices $\mathrm{C}$ and $\mathrm{D}$ respectively. Appendix $\mathrm{E}$ is devoted to a discussion of the uncertainties in energy-momentum measurements consistent with spacetime foam. In Appendix F we apply the results obtained in Appendix E to discuss high-energy $\gamma$ rays from distant gamma ray bursts and ultra-high energy cosmic ray events.

On notations, the subscript "P" denotes Planck units; thus $l_{P} \equiv\left(\hbar G / c^{3}\right)^{1 / 2} \sim 10^{-33} \mathrm{~cm}$ is the Planck length etc. On units, $k_{B}$ (the Boltzmann constant) and sometimes $\hbar$ and $\mathrm{c}$ are put equal to 1 for simplicity.

A note is in order: we are well aware of some critical papers that present a viewpoint that is contrary to some of the ideas contained in this paper. See, e.g., [10] and references contained therein.

\section{Quantum Fluctuations of Spacetime}

\subsection{Mapping the Geometry of Spacetime}

If spacetime indeed undergoes quantum fluctuations, the fluctuations will show up when we measure a distance $l$, in the form of uncertainties in the measurement. The question now is: how accurately can we measure this distance? Let us denote by $\delta l$ the accuracy with which we can measure $l$. We will also refer to $\delta l$ as the uncertainty or fluctuation of the distance $l$ for reasons that will become obvious shortly. One way to find $\delta l$ is to carry out a gedanken experiment to measure $l$. This is done in Appendix A. But, for later use, it is more convenient to find $\delta l$ by carrying out a process of mapping the geometry of spacetime. This method $[6,11]$ relies on the fact that quantum fluctuations of spacetime manifest themselves in the form of uncertainties in the geometry of spacetime. Hence the structure of spacetime foam can be inferred from the accuracy with which we can measure that geometry. Let us consider mapping out the geometry of spacetime for a spherical volume of radius $l$ over the amount of time $T=2 l / c$ it takes light to cross the volume. One way to do this is to fill the space with clocks, exchanging signals with other clocks and measuring the signals' times of arrival. This process of mapping the geometry of spacetime is a kind of computation, in which distances are gauged by transmitting and processing information. The total number of operations, including the ticks of the clocks and the measurements of signals, is bounded by the Margolus-Levitin theorem [12](see Appendix D) in quantum computation, which stipulates that the rate of operations for any computer cannot exceed the amount of energy $E$ that is available for computation divided by $\pi \hbar / 2$. A total mass $M$ of clocks then yields, via the MargolusLevitin theorem, the bound on the total number of operations given by $\left(2 M c^{2} / \pi \hbar\right) \times 2 l / c$. But to prevent black hole formation, $M$ must be less than $l c^{2} / 2 G$. Together, these two limits imply that the total number of operations that can occur in a spatial volume of radius $l$ for a time period $2 l / c$ is no greater than $2\left(l / l_{P}\right)^{2} / \pi$. To maximize spatial resolution, each clock must tick only once during the entire time period. The operations can be regarded as partitioning the spacetime volume into "cells", then on the average each cell occupies a spatial volume no less than $\left(4 \pi l^{3} / 3\right) /\left(2 l^{2} / \pi l_{P}^{2}\right)=2 \pi^{2} l l_{P}^{2} / 3$, yielding an average separation between neighhoring cells no less than $\left(2 \pi^{2} / 3\right)^{1 / 3} l^{1 / 3} l_{P}^{2 / 3}$. This spatial separation is interpreted as the average minimum uncertainty [13] in the measurement of a distance $l$, that is,

$$
\delta l \gtrsim l^{1 / 3} l_{P}^{2 / 3},
$$


where and henceforth (with a couple of exceptions) we drop multiplicative factors of order 1.

\subsection{Models of Spacetime Foam}

We can now understand why this quantum foam model has come to be known as the holographic model. Since, on the average, each cell occupies a spatial volume of $l l_{P}^{2}$, a spatial region of size $l$ can contain no more than $l^{3} /\left(l l_{P}^{2}\right)=\left(l / l_{P}\right)^{2}$ cells. Thus this model corresponds to the case of maximum number of bits of information $l^{2} / l_{P}^{2}$ in a spatial region of size $l$, that is allowed by the holographic principle [14] (see AppendixC).

To see this more concretely, consider a cubic region of space with linear dimension $l$. Conventional wisdom claims that the region can be partitioned into cubes as small as $\left(l_{P}\right)^{3}$. It follows that the number of degrees of freedom of the region is bounded by $\left(l / l_{P}\right)^{3}$, i.e., the volume of the region in Planck units. But conventional wisdom is wrong, for according to Eq. (1), the smallest cubes into which we can partition the region cannot have a linear dimension smaller than $\left(l l_{P}^{2}\right)^{1 / 3}$. Therefore, the number of degrees of freedom of the region is bounded by $\left[l /\left(l l_{P}^{2}\right)^{1 / 3}\right]^{3}$, i.e., the area of the region in Planck units, as stipulated by the holographic principle [14].

Recently Gambini and Pullin [15] have shown that holography follows from the framework of loop quantum gravity in spherical symmetry. They have derived from first principles an uncertainty in the determination of volumes that grows radially (corresponding to the $l$ above); i.e., they have recovered Eq. (1).

It will prove to be useful to compare the holographic model in the mapping of the geometry of spacetime with the one that corresponds to spreading the spacetime cells uniformly in both space and time. For the latter case, each cell has the size of $\left(l^{2} l_{P}^{2}\right)^{1 / 4}=l^{1 / 2} l_{P}^{1 / 2}$ both spatially and temporally so that each clock ticks once in the time it takes to communicate with a neighboring clock. Since the dependence on $l^{1 / 2}$ is the hallmark of a random-walk fluctuation, this quantum foam model corresponding to $\delta l \gtrsim\left(l l_{P}\right)^{1 / 2}$ is called the random-walk model [16]. Compared to the holographic model, the randomwalk model predicts a coarser spatial resolution, i.e., a larger distance fluctuation, in the mapping of spacetime geometry. It also yields a smaller bound on the information content in a spatial region, viz., $\left(l / l_{p}\right)^{2} /\left(l / l_{P}\right)^{1 / 2}=\left(l^{2} / l_{P}^{2}\right)^{3 / 4}=\left(l / l_{P}\right)^{3 / 2}$ bits.

Actually there are many other models of spacetime foam, in addition to the holographic model and the random-walk model. We can parametrize them according to $\delta l \sim l^{1-\alpha} l_{P}^{\alpha}$ for $\alpha$ of order 1 , with $\alpha=2 / 3$ and $1 / 2$ for the holographic model and the random-walk model respectively [17].

Note that the minimum $\delta l$ just found for the holographic model corresponds to the case of maximum energy density $\rho=(3 / 8 \pi)\left(l l_{P}\right)^{-2}$ for a sphere of radius $l$ not to collapse into a black hole. Hence the holographic model, unlike the other models, requires, for its consistency, the energy density to have the critical value. By contrast, for instance, the random-walk model corresponds to an energy density $\left(l l_{P}\right)^{-2} \gtrsim \rho \gtrsim l^{-5 / 2} l_{P}^{-3 / 2}$. (The upper bound corresponds to the clocks ticking every $\left(l l_{P}\right)^{1 / 2}$ while the lower bound corresponds to the clocks ticking only once during the entire time $2 l / c$.) 


\subsection{Cumulative Effects of Spacetime Fluctuations}

Let us examine the cumulative effects [18] of spacetime fluctuations over a large distance. Consider a distance $l$, and divide it into $l / \lambda$ equal parts each of which has length $\lambda$ (that can be as small as $l_{P}$ ). If we start with $\delta \lambda$ from each part, the question is how do the $l / \lambda$ parts add up to $\delta l$ for the whole distance $l$. In other words, we want to find the cumulative factor $C$ defined by $\delta l=C \delta \lambda$. Since $\delta l \sim l^{1 / 3} l_{P}^{2 / 3}=l_{P}\left(l / l_{P}\right)^{1 / 3}$ and $\delta \lambda \sim \lambda^{1 / 3} l_{P}^{2 / 3}=l_{P}\left(\lambda / l_{P}\right)^{1 / 3}$, the result is $C=(l / \lambda)^{1 / 3}$. Note that the cumulative factor is not linear in $(l / \lambda)$, i.e., $\frac{\delta l}{\delta \lambda} \neq \frac{l}{\lambda}$. (In general, it is much smaller than $l / \lambda$ ). The reason for this is obvious: the $\delta \lambda$ 's (which take on \pm sign with equal probability) from the $l / \lambda$ parts in $l$ do not add coherently. In general, for spacetime foam models corresponding to $\delta l \sim l^{1-\alpha} l_{P}^{\alpha}$, the cumulative factor is given by $C=(l / \lambda)^{1-\alpha}$. Thus for the random-walk model, the cumulative factor is $(l / \lambda)^{1 / 2}$. Note that for the holographic case, the individual fluctuations cannot be completely random (as opposed to the random-walk model); strangely successive fluctuations appear to be entangled and somewhat anti-correlated (i.e., a plus fluctuation is slightly more likely followed by a minus fluctuation and vice versa), in order that together they produce a total fluctuation less than that in the random-walk model. This small amount of anti-correlation between successive fluctuations (corresponding to what statisticians call fractional Brownian motion with self-similarity parameter $\frac{1}{3}$ ) must be due to quantum gravity effects.

On the other hand, if successive fluctuations are completely anti-correlated, then the fluctuation of a distance $l$ is given by the minuscule $l_{P}$, independent of the size of the distance. For completeness, we mention that a priori there are also models with positive correlations between successive fluctuations. But these models yield unacceptably large fluctuations in distance measurements - it turns out (see next section) that these models (actually all models with $\alpha \lesssim 0.6$ corresponding to the hatched line in Fig. 1) have already been observationally ruled out.

Figure 1. Lower bounds on $\delta l$ for the various quantum gravity models. The fluctuation of a distance $l$ is given by the sum of $l / l_{P}$ fluctuations each by plus or minus $l_{P}$. Spacetime foam appears to choose a small anti-correlation (i.e., negative correlation) between successive fluctuations, giving a cube root dependence in the number $l / l_{p}$ of fluctuations for the total fluctuation of $l$ (indicated by the arrow). The corresponding model falls between the two extreme cases of complete randomness, i.e., zero correlation (corresponding to $\delta l \sim l^{1 / 2} l_{P}^{1 / 2}$ ) and complete anti-correlation (corresponding to $\delta l \sim l_{P}$ ).

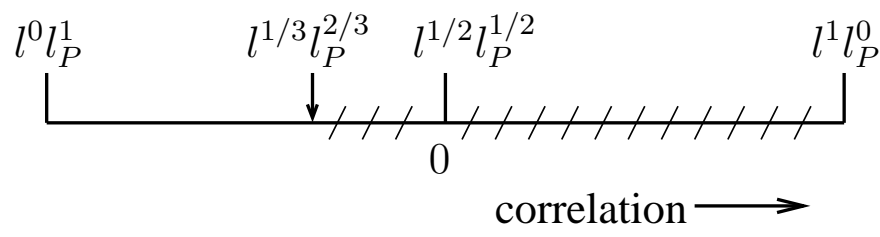

\section{Probing Quantum Foam with Extragalactic Sources}

The Planck length $l_{P} \sim 10^{-33} \mathrm{~cm}$ is so short that we need an astronomical (even cosmological) distance $l$ for its fluctuation $\delta l$ to be detectable. Let us consider light (with wavelength $\lambda$ ) from distant 
quasars or bright active galactic nuclei $[18,19]$. Due to the quantum fluctuations of spacetime, the wavefront, while planar, is itself "foamy", having random fluctuations in phase [18] $\Delta \phi \sim 2 \pi \delta l / \lambda$ and in the direction of the wave vector [7] given by $\Delta \phi / 2 \pi$ (for $\delta l \ll \lambda$ ). In effect, spacetime foam creates a "seeing disk" whose angular diameter is $\sim \Delta \phi / 2 \pi$. For an interferometer with baseline length $D$, this means that dispersion will be seen as a spread in the angular size of a distant point source, causing a reduction in the fringe visibility when $\Delta \phi / 2 \pi \sim \lambda / D$. For a quasar of $1 \mathrm{Gpc}$ away, at infrared wavelength, the holographic model predicts a phase fluctuation $\Delta \phi \sim 2 \pi \times 10^{-9}$ radians. On the other hand, an infrared interferometer (like the Very Large Telescope Interferometer) with $D \sim 100$ meters has $\lambda / D \sim 5 \times 10^{-9}$. Thus, in principle, this method will allow the use of interferometry fringe patterns to test the holographic model! Furthermore, these tests can be carried out without guaranteed time using archived high resolution, deep imaging data on quasars, and possibly, supernovae from existing and upcoming telescopes.

The key issue here is the sensitivity of the interferometer. The lack of observed fringes may simply be due to the lack of sufficient flux (or even just effects originated from the turbulence of the Earth's atmosphere) rather than the possibility that the instrument has resolved a spacetime foam generated halo. But, given sufficient sensitivity, the VLTI, for example, with its maximum baseline, presumably has sufficient resolution to detect spacetime foam halos for low redshift quasars, and in principle, it can be even more effective for the higher redshift quasars. Note that the test is simply a question of the detection or non-detection of fringes. It is not a question of mapping the structure of the predicted halo.

In the meantime, we can use existing archived data on quasars or active galactic nuclei from the Hubble Space Telescope to test the quantum foam models [7]. Consider the case of PKS1413+135 [20], an AGN for which the redshift is $z=0.2467$. With $l \approx 1.2 \mathrm{Gpc}$ and $\lambda=1.6 \mu \mathrm{m}$, we [18] find $\Delta \phi \sim 10 \times 2 \pi$ and $10^{-9} \times 2 \pi$ for the random-walk model and the holographic model of spacetime foam respectively. With $D=2.4 \mathrm{~m}$ for HST, we expect to detect halos if $\Delta \phi \sim 10^{-6} \times 2 \pi$. Thus, the HST image only fails to test the holographic model by 3 orders of magnitude.

However, the absence of a quantum foam induced halo structure in the HST image of PKS1413+135 rules out convincingly the random-walk model. (In fact, the scaling relation discussed above indicates that all spacetime foam models with $\alpha \lesssim 0.6$ are ruled out by this HST observation.) As we will see in the next section, this result has profound implications for cosmology [6-9].

\section{From Quantum Foam to (Holographic Foam) Cosmology}

We can draw a useful conclusion [6-9] from the observed cosmic critical density in the present era (consistent with the prediction of the cosmology based on the holographic model of spacetime foam, which henceforth we call the holographic foam cosmology (HFC)) $\rho \sim H_{0}^{2} / G \sim\left(R_{H} l_{P}\right)^{-2}$ (about 10 joule per cubic meter), where $H_{0}$ is the present Hubble parameter of the observable universe and $R_{H}$ is the Hubble radius. Treating the whole universe as a computer[6, 21], one can apply the Margolus-Levitin theorem to conclude that the universe computes at a rate $\nu$ up to $\rho R_{H}^{3} \sim R_{H} l_{P}^{-2}$ ( $\sim 10^{106} \mathrm{op} / \mathrm{sec}$ ), for a total of $\left(R_{H} / l_{P}\right)^{2}\left(\sim 10^{122}\right)$ operations during its lifetime so far. If all the information of this huge computer is stored in ordinary matter, then we can apply standard methods of statistical mechanics to find that the total number $I$ of bits is $\left[\left(R_{H} / l_{P}\right)^{2}\right]^{3 / 4}=\left(R_{H} / l_{P}\right)^{3 / 2}\left(\sim 10^{92}\right)$. It follows that each bit flips once in the amount of time given by $I / \nu \sim\left(R_{H} l_{P}\right)^{1 / 2}\left(\sim 10^{-14} \mathrm{sec}\right)$. On the other hand, the 
Table 1. Random-walk model versus holographic model. The corresponding quantities for the random-walk model (second row) and the holographic model (third row) of spacetime foam (STF) appear in the same columns in the following Table. (Entropy is measured in Planck units.)

\begin{tabular}{|c|c|c|c|c|c|}
\hline $\begin{array}{c}\text { STF } \\
\text { model }\end{array}$ & $\begin{array}{c}\text { distance } \\
\text { fluctuations }\end{array}$ & $\begin{array}{c}\text { entropy } \\
\text { bound }\end{array}$ & $\begin{array}{c}\text { energy } \\
\text { density }\end{array}$ & $\begin{array}{c}\text { matter/ } \\
\text { energy }\end{array}$ & $\begin{array}{c}\text { type of } \\
\text { statistics }\end{array}$ \\
\hline \hline $\begin{array}{c}\text { random- } \\
\text { walk }\end{array}$ & $\delta l \gtrsim l^{1 / 2} l_{P}^{1 / 2}$ & $(A r e a)^{3 / 4}$ & $\begin{array}{c}\left(l l_{P}\right)^{-2} \gtrsim \rho \\
\gtrsim l^{-5 / 2} l_{P}^{-3 / 2}\end{array}$ & ordinary & $\begin{array}{c}\text { bose } / \\
\text { fermi }\end{array}$ \\
\hline \hline $\begin{array}{c}\text { holo- } \\
\text { graphic }\end{array}$ & $\delta l \gtrsim l^{1 / 3} l_{P}^{2 / 3}$ & Area & $\rho \lesssim\left(l l_{P}\right)^{-2}$ & $\begin{array}{c}\text { dark } \\
\text { energy }\end{array}$ & infinite \\
\hline
\end{tabular}

average separation of neighboring bits is $\left(R_{H}^{3} / I\right)^{1 / 3} \sim\left(R_{H} l_{P}\right)^{1 / 2}\left(\sim 10^{-3} \mathrm{~cm}\right)$. Hence, assuming only ordinary matter exists to store all the information in the universe results in the conclusion that the time to communicate with neighboring bits is equal to the time for each bit to flip once. It follows that the accuracy to which ordinary matter maps out the geometry of spacetime corresponds exactly to the case of events spread out uniformly in space and time discussed above for the case of the random-walk model of spacetime foam.

But, as shown in the previous section, the sharp images of distant quasars or active galactic nuclei observed at the Hubble Space Telescope have ruled out the random-walk model. From the demise of the random-walk model and the fact that ordinary matter only contains an amount of information dense enough to map out spacetime at a level consistent with the random-walk model, one can now infer that spacetime must be mapped to a finer spatial accuracy than that which is possible with the use of ordinary matter. But if ordinary matter does not do, there must be another kind of substance with which spacetime can be mapped to the observed accuracy, conceivably as given by the holographic model. The natural conclusion [7-9] is that unconventional (dark [22]) energy/matter exists! Note that this argument does not make use of the evidence from recent cosmological (supernovae, cosmic microwave background, and galaxy clusters) observations [23].

A comparison between what the random-walk model and the holographic model yield for the entropy bound etc. is given in Table 1. See the next section for the explanation of the last column.

The fact that our universe is observed to be at or very close to its critical density must be taken as solid albeit indirect evidence in favor of the holographic model because, as discussed above, it is the only model that requires, for its consistency, the maximum energy density without causing gravitational collapse. Specifically, according to the HFC, the cosmic density is

$$
\rho=(3 / 8 \pi)\left(R_{H} l_{P}\right)^{-2} \sim\left(H / l_{P}\right)^{2}
$$

and the cosmic entropy is given by

$$
I \sim\left(R_{H} / l_{P}\right)^{2}
$$

Thus the average energy carried by each bit is $\rho R_{H}^{3} / I \sim R_{H}^{-1}\left(\sim 10^{-31} \mathrm{eV}\right)$. Such long-wavelength (hence "non-local") bits or "particles" carry negligible kinetic energy. Also according to HFC, it takes each unconventional bit the amount of time $I / \nu \sim R_{H}$ to flip. Thus, on the average, each bit flips once over the course of cosmic history. Compared to the conventional bits carried by ordinary matter, 
these bits are rather passive and inert (which, by the way, may explain why dark energy is dark). This is understandable since each unconventional bit has, at its disposal, only such a minuscule amount of energy. But together (there can be as many as $\left(R_{H} / l_{P}\right)^{2} \sim 10^{123}$ of them in the present observable universe, far outnumbering the $10^{92}$ or so particles of ordinary matter and radiation), they supply the missing mass/energy of the universe. Accelerating the cosmic expansion is a relatively simple task, computationally speaking.

\section{Infinite Statistics and Nonlocality}

\subsection{Infinite Statistics}

What is the overriding difference between conventional matter and unconventional energy/matter (i.e., dark energy and perhaps also dark matter)? To find that out, let us first consider a perfect gas of $N$ particles obeying Boltzmann statistics (which, rigorously speaking, is not a physical statistics but is still a useful statistics to work with) at temperature $T$ in a volume $V$. For the problem at hand, as the lowest-order approximation, we can neglect the contributions from matter and radiation to the cosmic energy density for the recent and present eras. Then the Friedmann equations for $\rho \sim H^{2} / G$ can be solved by $H \propto 1 / a$ and $a \propto t$, where $a(t)$ is the cosmic scale factor. Thus let us take $V \sim R_{H}^{3}$, $T \sim R_{H}^{-1}$, and $N \sim\left(R_{H} / l_{P}\right)^{2}$. A standard calculation (for the relativistic case) yields the partition function $Z_{N}=(N !)^{-1}\left(V / \lambda^{3}\right)^{N}$, where $\lambda=(\pi)^{2 / 3} / T$. With the free energy given by $F=-T \ln Z_{N}=$ $-N T\left[\ln \left(V / N \lambda^{3}\right)+1\right]$, we get, for the entropy of the system,

$$
S=-(\partial F / \partial T)_{V, N}=N\left[\ln \left(V / N \lambda^{3}\right)+5 / 2\right] .
$$

For the non-relativistic case with the effective mass $m \sim R_{H}^{-1}$ (coming from some sort of potential with which we are not going to concern ourselves), the only changes in the above expressions are given by the substitution $\lambda \longrightarrow(2 \pi / m T)^{1 / 2}$. With $m \sim T \sim R_{H}^{-1}$, there is no significant qualitative difference between the non-relativistic and relativistic cases.

The important point to note is that, since $V \sim \lambda^{3}$, the entropy $S$ in Eq. (4) becomes nonsensically negative unless $N \sim 1$ which is equally nonsensical because $N$ should not be too different from $\left(R_{H} / l_{P}\right)^{2} \gg 1$. Intentionally we have calculated the entropy by employing the familiar Boltzmann statistics (with the correct Boltzmann counting factor), only to arrive at a contradictory result. But now the solution to this contradiction is pretty obvious: the $N$ inside the log in Eq. (4) somehow must be absent. Then $S \sim N \sim\left(R_{H} / l_{P}\right)^{2}$ without $N$ being small (of order 1 ) and $\mathrm{S}$ is non-negative as physically required. That is the case if the "particles" are distinguishable and nonidentical! For in that case, the Gibbs $1 / N$ ! factor is absent from the partition function $Z_{N}$, and the entropy becomes

$$
S=N\left[\ln \left(V / \lambda^{3}\right)+3 / 2\right]
$$

Now the only known consistent statistics in greater than two space dimensions without the Gibbs factor (recall that the Fermi statistics and Bose statistics give similar results as the conventional Boltzmann statistics at high temperature) is infinite statistics (sometimes called "quantum Boltzmann statistics") [24-26]. Thus we have shown that the "particles" constituting dark energy obey infinite statistics, 
instead of the familiar Fermi or Bose statistics [9]. What is infinite statistics? Succinctly, a Fock realization of infinite statistics is provided by a $q$ deformation of the commutation relations of the oscillators: $a_{k} a_{l}^{\dagger}-q a_{l}^{\dagger} a_{k}=\delta_{k l}$ with $q$ between -1 and 1 (the case $q= \pm 1$ corresponds to bosons or fermions). States are built by acting on a vacuum which is annihilated by $a_{k}$. Two states obtained by acting with the $N$ oscillators in different orders are orthogonal. It follows that the states may be in any representation of the permutation group. The statistical mechanics of particles obeying infinite statistics can be obtained in a way similar to Boltzmann statistics, with the crucial difference that the Gibbs $1 / N$ ! factor is absent for the former. Infinite statistics can be thought of as corresponding to the statistics of identical particles with an infinite number of internal degrees of freedom, which is equivalent to the statistics of nonidentical particles since they are distinguishable by their internal states.

\subsection{Nonlocality}

Infinite statistics appears to have one "defect": a theory of particles obeying infinite statistics cannot be local [26-28]. (That is, the fields associated with infinite statistics are not local, neither in the sense that their observables commute at spacelike separation nor in the sense that their observables are pointlike functionals of the fields.) The expression for the number operator (for the case of $q=0$ )

$$
n_{i}=a_{i}^{\dagger} a_{i}+\sum_{k} a_{k}^{\dagger} a_{i}^{\dagger} a_{i} a_{k}+\sum_{l} \sum_{k} a_{l}^{\dagger} a_{k}^{\dagger} a_{i}^{\dagger} a_{i} a_{k} a_{l}+\ldots
$$

is both nonlocal and nonpolynomial in the field operators, and so is the Hamiltonian. The lack of locality may make it difficult to formulate a relativistic verion of the theory; but it appears that a non-relativistic theory can be developed. Lacking locality also means that the familiar spin-statistics relation is no longer valid for particles obeying infinite statistics; hence they can have any spin. Remarkably, the TCP theorem and cluster decomposition have been shown to hold despite the lack of locality [26].

Actually the lack of locality for theories of infinite statistics may have a silver lining. According to the holographic principle, the number of degrees of freedom in a region of space is bounded not by the volume but by the surrounding surface. This suggests that the physical degrees of freedom are not independent but, considered at the Planck scale, they must be infinitely correlated, with the result that the spacetime location of an event may lose its invariant significance. Since the holographic principle is believed to be an important ingredient in the formulation of quantum gravity, the lack of locality for theories of infinite statistics may not be a defect; it can actually be a virtue. Perhaps it is this lack of locality that makes it easier to incorporate gravitational interactions in the theory. Quantum gravity and infinite statistics appear to fit together nicely, and nonlocality seems to be a common feature of both of them [9].

We note the following related work. Using the Matrix theory approach, Jejjala, Kavic and Minic [29] have argued that dark energy quanta obey infinite statistics (and that the fine structure of dark energy is governed by a Wien distribution). They have also concluded that the non-locality present in systems obeying infinite statistics and the non-locality present in holographic theories may be related.

Strominger [30] has shown that the wave function of many similarly charged extremal black holes depends on each black hole's position, and thus the black holes can be considered as distinguishable and accordingly obey infinite statistics. 
Figure 2. Connecting the different ideas.

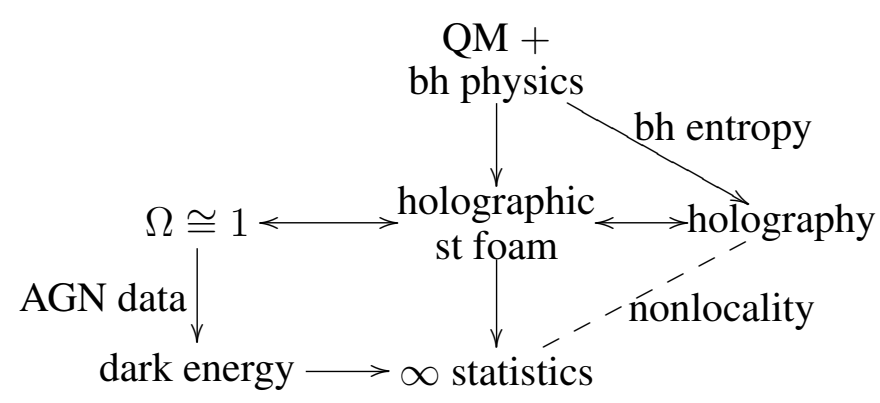

More generally Giddings [31] has observed that the non-perturbative dynamics of gravity is nonlocal. His argument is based on several reasons: lack of a precise definition in quantum gravity -connected with the apparent absence of local observables; indications from high-energy gravitational scattering; hints from string theory, particularly the AdS/CFT correspondence; conundrums of quantum cosmology; and the black hole information paradox. Horowitz [32] has noted that quantum gravity may need some violation of locality; thus when one reconstructs the string theory from the gauge theory (in the AdS/CFT correspondence), physics may not be local on all length scales. Meanwhile Ahluwalia [33] has argued that when measurement processes involve energies of the order of the Planck scale, the fundamental assumption of locality may no longer be a good approximation. He has shown that position measurements alter the spacetime metric in a fundamental manner and this unavoidable change in the spacetime metric destroys the commutativity (and hence locality) of position measurement operators.

\section{Summary, Discussion and Conclusion}

A short summary of this review article is given by the accompanying flow chart Figure 2.

Starting from quantum mechanics and some rudimentary black hole physics (viz, there is an upper bound of matter/energy that can be put in a spatial region without the region collapsing into a black hole), we derive the holographic model of spacetime foam. From the simple observation that the cosmic energy density is critical (i.e., the density parameter of the universe $\Omega \cong 1$ ), aided by some archived data on quasar or AGN from the Hubble Space Telescope, we are led to conclude that dark energy exists. This observation is a solid piece of evidence in favor of the holographic foam model which, after all, "predicts" that the cosmic energy density has the critical value. One is then invited to apply the holographic model to cosmology, leading to the logical speculation that the constituents of dark energy obey infinite statistics. We have now come full circle, starting with holography and ending with infinite statistics, with both sharing one common property: nonlocality. In the whole discussion, entropy plays a leading role, first in motivating holography, and secondly in determining, via its positivity property, that the "particles" constituting dark energy obey infinite statistics.

We conclude with some observation and a partial list of open questions.

(1) We have considered a perfect gas consisting of "particles" of extremely long wavelength, obeying Boltzmann statistics in the Universe at temperature $T$. But we have seen that these "particles" have had interactions only of order one time on the average during the entire cosmic history. A question can be 
raised as to whether such an inert gas can come to thermal equilibrium at any well defined temperature. We do not have a good answer; but the fact that all these "particles", though extremely inert, have a wavelength comparable to the observable Hubble radius may mean that they overlap significantly, and accordingly can perhaps share a common temperature.

(2) These "particles" provide a (more or less) spatially uniform energy density, like a time-dependent cosmological constant [34]. But in a way, this type of models is preferrable to the cosmological constant because it may be easier to understand a zero cosmological constant (perhaps due to a certain not-yetunderstood symmetry or initial condition [35]) than an exceedingly small (but non-zero) cosmological constant.

(3) Recall that earlier cosmic epochs are associated with $\rho \propto a^{-4}$ (radiation-domination) and (followed by) $\rho \propto a^{-3}$ (matter-domination). In a scenario of the holographic foam cosmology we are pursuing, these epochs are succeeded by the dark-energy-dominated era with $\rho \propto a^{-2}$. We note that the successes of the conventional big bang cosmology, such as the primordial nucleosynthesis, are not affected by this form of dark energy.

(4) An obvious question concerns the sign of the pressure for the gas of dark energy "particles". For cosmic energy density $\rho \sim H^{2} / G$ with $H \propto 1 / a$, the equation of state is given by $w=p / \rho \sim$ $-1 / 3$, not negative enough to give an accelerating expansion. However, it has been pointed out by Zimdahl and Pavon [36] that a transition from an earlier decelerating to a recent and present accelerating cosmic expansion can arise as a pure interaction phenomenon if pressureless dark matter is coupled to holographic dark energy with $\rho \propto H^{2}$ [37]. As a bonus, within the framework of such cosmological models, we can now understand why, in addition to dark energy, dark matter has to exist. On the other hand, this scenario will become less natural if the equation of state $w$ turns out to be very close to -1 .

(5) Critical cosmic energy density is the hallmark of the inflationary paradigm [38]. Can the holographic foam cosmology (which requires $\Omega \cong 1$ ) supplement inflation in solving cosmological problems and in providing the necessary primordial perturbations to yield the observed astronomical structures? What are the phenomenological consequences of HFC?

(6) While it appears quite reasonable that holography (and quantum gravity in general) and infinite statistics are compatible, exactly how they are related is not yet clear. In particular, how is the nonlocality in holography related to the nonlocality present in theories with infinite statistics? (We venture a guess: Entropy is the common link, so it may hold the key in understanding the relation.)

Before last century, spacetime was regarded as nothing more than a passive and static arena in which events took place. Early last century, Einstein's general relativity changed that viewpoint and promoted spacetime to an active and dynamical entity. Quantum mechanics blossomed around that time. But the challenge to understand the quantum nature of spacetime was not taken up seriously until quite a bit later. Now many physicists believe that spacetime, like all matter and energy, undergoes quantum fluctuations. These quantum fluctuations make spacetime foamy on small spacetime scales. In this article, we have used a global positioning-like system to measure the geometry of spacetime, to show that spacetime fluctuations, on the average, scale as the cube root of distances. This result is very general, depending only on quantum mechanics and limited black hole physics (viz., the size of a black hole scales linearly with its mass). The cube root dependence is strange, but it has been shown to be consistent with the holographic principle and (as shown in Appendix B) semi-classical black hole physics in general. 
Furthermore, applied to cosmology, it successfully "predicts" the existence of dark energy and that the cosmic energy density is critical. To the author at least, the cube-root result for spacetime foam is as beautiful as it is strange - and, when the Very Large Telescope Interferometer reaches its design performance, it may even be proven to be true.

\section{Acknowledgments}

This work was supported in part by the US Department of Energy and the Bahnson Fund of the University of North Carolina. I thank H. van Dam, G. Amelino-Camelia, W. Christiansen, T. W. Kephart, O. W. Greenberg, M. Arzano, J. Pullin, D. Minic, L. Mersini, R. Rohm, X. Calmet, S. Hsu, T. Biswas, N. Mavromatos, S. Giddings, V. Husain, D. Pavon, and W. Zimdahl for useful discussions, L. L. Ng for his help in the preparation of this manuscript, and P. Zizzi for her invitation to write this review article. I dedicate this paper to the late John Wheeler who kindly took an interest in my work, and also to my wife Yvonne Woon-yu who is blessed with a keen eye for things of strange beauty.

\section{Appendix A: Salecker \& Wigner's Gedanken Experiment}

Let us conduct a thought experiment to measure a distance $l$. Following Salecker $\&$ Wigner [39] we can put a clock (of mass $m$ ) at one end of the distance and a mirror at the other end. By sending a light signal from the clock to the mirror in a timing experiment, we can determine the distance $l$. But the clock's position jiggles according to Heisenberg's uncertainty principle in quantum mechanics, resulting in an uncertainty in the measurement:

$$
\delta l^{2} \gtrsim \frac{\hbar l}{m c} .
$$

The jiggling of the clock is reduced by using a massive clock. But, according to general relativity, a massive clock would distort the surrounding space severely, affecting adversely the accuracy in the measurement of the distance:

$$
\delta l \gtrsim \frac{G m}{c^{2}} .
$$

The conflicting requirements from quantum mechanics and general relativity reach a compromise, resulting in the elimination of the dependence on $m$, and yielding $[2,4,5]$ (by taking the product of Eq. (8) and Eq. (7)):

$$
\delta l \gtrsim\left(l l_{P}^{2}\right)^{1 / 3}=l_{P}\left(\frac{l}{l_{P}}\right)^{1 / 3} .
$$

Obviously the accuracy of distance measurements is intrinsically limited by this amount of uncertainty or fluctuation.

\section{Appendix B: From Space-time Fluctuations to Black Holes}

To gain confidence in the strange scaling of space-time fluctuations with the cube-root of distances, let us look for theoretical "evidence". Fortunately such circumstantial evidence does exist — in the sector of black hole physics. To show that, we have to make a small detour to consider clocks and computers $[5,40]$ first. 
Table 2. Distance measurements, clocks and computers. The corresponding quantities in the discussion of distance measurements (first column), clocks (second column), and computers (third column) appear in the same rows in the following Table.

\begin{tabular}{||c||c||c||}
\hline \hline $\begin{array}{c}\text { distance } \\
\text { measurements }\end{array}$ & clocks & computers \\
\hline \hline$\delta l / c$ & $t$ & $1 / \nu$ \\
\hline$l / c$ & $T$ & $I / \nu$ \\
\hline$\delta l^{2} \gtrsim \hbar l / m c$ & $t^{2} \gtrsim \hbar T / m c^{2}$ & $I \nu \lesssim m c^{2} / \hbar$ \\
\hline$\delta l \gtrsim G m / c^{2}$ & $t \gtrsim G m / c^{3}$ & $\nu \lesssim c^{3} / G m$ \\
\hline$l /(\delta l)^{3} \lesssim l_{P}^{-2}\left(\delta l \gtrsim l^{1 / 3} l_{P}^{2 / 3}\right)$ & $T / t^{3} \lesssim t_{P}^{-2}$ & $I \nu^{2} \lesssim t_{P}^{-2}=c^{5} / \hbar G$ \\
\hline \hline
\end{tabular}

\subsection{Clocks}

Consider a clock (of mass $m$ ), capable of resolving time to an accuracy of $t$, for a period of time $T$. Then bounds on the resolution time and the running time of the clock can be derived by following an argument very similar to that used above in the analysis of the gedanken experiment to measure distances. Actually, the two arguments are so similar that one can identify the corresponding quantities. [See Table 2.]

Following the argument in the previous Appendix, we obtain

$$
t^{2} \gtrsim \frac{\hbar T}{m c^{2}}, \quad t \gtrsim \frac{G m}{c^{3}}, \quad t \gtrsim t_{P}\left(\frac{T}{t_{P}}\right)^{1 / 3},
$$

the analogs of Eq. (7), Eq. (8) and Eq. (9) respectively. The last inequality yields a fundamental limit on how accurate a clock can be in measuring a given amount of time $T$. Recently Gambini, Porto and Pullin [41] have applied this result to argue that, since one cannot have a perfectly classical clock in nature, quantum mechanics needs to be modified in that quantum evolution is not unitary. Such a non-unitary evolution leads to potentially observable decoherence, e.g., in a system of Bose-Einstein condensates. These authors have further claimed that, in real life, one could never observe the black hole information paradox, since quantum states decohere (due to our lack of perfect clocks) at a rate faster than the one an evaporating black hole makes it disappear.

\subsection{Computers}

We can easily translate the above relations for clocks into useful relations for a simple computer. Since the resolution time $t$ for clocks is the smallest time interval relevant in the problem, the fastest possible processing frequency is given by its reciprocal, i.e., $1 / t$. Thus if $\nu$ denotes the clock rate of the computer, i.e., the number of operations per bit per unit time, then it is natural to identify $\nu$ with $1 / t$. To identify the number $I$ of bits of information in the memory space of a simple computer, we recall that the running time $T$ is the longest time interval relevant in the problem. Thus, the maximum number of steps of information processing is given by the running time divided by the resolution time, i.e., $T / t$. It follows that one can identify the number $I$ of bits of the computer with $T / t$. (One can think of a tape of length $c T$ as the memory space, partitioned into bits each of length $c t$.) The bounds on the precision and lifetime of a clock given by Eq. (10) are now translated into bounds on the rate of computation and 
number of bits in the computer, yielding respectively

$$
I \nu \lesssim \frac{m c^{2}}{\hbar}, \quad \nu \lesssim \frac{c^{3}}{G m}, \quad I \nu^{2} \lesssim \frac{c^{5}}{\hbar G} .
$$

The first inequality shows that the speed of computation is bounded by the energy of the computer divided by $\hbar$, in agreement with the Margolus-Levitin theorem (see Appendix D).

\subsection{Black Holes}

Now we can apply what we have learned about clocks and computers to black holes of mass $m$ [5, 40]. Let us consider using a black hole to measure time. It is reasonable to use the light travel time around the black hole's horizon as the resolution time of the clock, i.e., $t \sim G m / c^{3} \equiv t_{B H}$, then from the first equation in Eq. (10), one immediately finds that

$$
T \sim \frac{G^{2} m^{3}}{\hbar c^{4}} \equiv T_{B H}
$$

which is the celebrated Hawking black hole lifetime.

Finally, let us consider using a black hole to do computations [5, 6, 21, 40]. This may sound like a ridiculous proposition. But if we believe that black holes evolve according to quantum mechanical laws, it is possible, at least in principle, to program black holes to perform computations that can be read out of the fluctuations in the Hawking black hole radiation. How large is the memory space of a black hole computer, and how fast can it compute? Applying the results for computation derived above, we readily find the number of bits in the memory space of a black hole computer, given by the lifetime of the black hole divided by its resolution time as a clock, to be

$$
I=\frac{T_{B H}}{t_{B H}} \sim \frac{m^{2}}{m_{P}^{2}} \sim \frac{r_{S}^{2}}{l_{P}^{2}}
$$

where $m_{P}=\hbar /\left(t_{P} c^{2}\right)$ is the Planck mass, and $r_{S}^{2}$ denotes the event horizon area of the black hole. This gives the number $I$ of bits as the event horizon area in Planck units, in agreement with the identification of black hole entropy. (Note that Eq. (13) can also be derived from Eq. (11).) Furthermore, the number of operations per unit time for a black hole computer is given by $I \nu=\left(T_{B H} / t_{B H}\right) \times\left(1 / t_{B H}\right) \sim m c^{2} / \hbar$, viz., its energy divided by Planck's constant. (It is interesting that all the bounds on clocks and computation discussed above are actually saturated by black hole clocks and black hole computers respectively.) Thus it is now abundantly clear that the graininess of space-time due to quantum fluctuations that we found above is entirely consistent with well-known black hole physics.

We conclude this Appendix with a speculation on the nature of black hole microstates. [42] It is baffling that ordinary matter configurations on the verge of becoming black holes have entropy $S \sim$ $(\text { Area })^{3 / 4}$ whereas the black holes themselves have entropy $S \sim$ Area. What can account for this difference in the amount of entropy? Inspired by the columns under the headings of "entropy bound" and "type of statistics" in Table 1, we make the following conjecture. While ordinary matter obeys bose or fermi statistics, it is possible that the microscopic constituents of black holes effectively obey infinite statistics. To wit, to a black hole of size $r_{S}$ and Hawking temperature $\sim 1 / r_{S}$, the application of Eq. (5) readily yields $S \sim\left(r_{S} / l_{P}\right)^{2}$ by taking the number of microscopic constituents to be $N \sim\left(r_{S} / l_{P}\right)^{2}$. 
(On the other hand, we do not see how Eq. (4) can give an entropy that scales as the area $r_{S}^{2}$.) That said, it is hard to show how matter obeying bose or fermi statistics can become constituents obeying infinite statistics upon gravitational collapse to form a black hole.

\section{Appendix C: Holographic Principle}

In essence, the holographic principle[14] stipulates that although the world around us appears to have three spatial dimensions, its contents can actually be encoded on a two-dimensional surface, like a hologram. In other words, the maximum entropy of a region of space is given (aside from multiplicative factors of order 1 which we ignore as we have so far) by its surface area in Planck units. This result can be derived by appealing to black hole physics and the second law of theromodynamics as follows [14]. Consider a system with entropy $S_{0}$ inside a spherical region $\Gamma$ bounded by surface area $A$. Its mass must be less than that of a black hole with horizon area $A$ (otherwise it would have collapsed into a black hole). Now imagine a spherically symmetric shell of matter collapsing onto the original system with just the right amount of energy so that together with the original mass, it forms a black hole which just fills the region $\Gamma$. The black hole so formed has entropy $S \sim A / l_{P}^{2}$. But according to the second law of thermodynamics, $S_{0} \leq S$. It follows immediately that $S_{0} \lesssim A / l_{P}^{2}$, and hence the maximum entropy of a region of space is bounded by its surface area, as asserted by the holographic principle.

\section{Appendix D: the Margolus-Levitin Theorem}

The Margolus-Levitin theorem [12] plays an important role in our discussion in the text. For the sake of completeness, in this Appendix we follow Margolus and Levitin to derive it. Consider the maximum speed of dynamical evolution for a given physical system. Assume that the system has a discrete energy spectrum $E_{n}, n=0,1,2 \ldots$ with the lowest energy chosen to be $E_{0}=0$. We can write an arbitrary state $|\psi\rangle$ as a superposition of energy eigenstates, with coefficients $c_{n}$ at time $t=0$. Let $\left|\psi_{0}\right\rangle$ evolve for a time $t$ to become $\left|\psi_{t}\right\rangle$. Denote the transition amplitude $\left\langle\psi_{0} \mid \psi_{t}\right\rangle$ by $S(t)$. We want to find the smallest value of $t$ such that $S(t)=0$. To do that, we note that $\operatorname{Re}(S)=\sum_{n}\left|c_{n}\right|^{2} \cos \left(E_{n} t / \hbar\right)$. Using the inequality $\cos x \geq 1-(2 / \pi)(x+\sin x)$, valid for $x \geq 0$, we get $\operatorname{Re}(S) \geq 1-2 E t /(\pi \hbar)+2 \operatorname{Im}(S) / \pi$, where $E$ denotes the average energy of the system. But $S(t)=0$ implies both $\operatorname{Re}(S)=0$ and $\operatorname{Im}(S)=0$. So this inequality becomes $0 \geq 1-4 E t / h$, where $h=2 \pi \hbar$. Thus the earliest that $S(t)$ can possibly equal zero is when $t=h / 4 E$. Applied to a computer, this result implies that the maximum speed of computation is given by $4 / h$ times the energy available for computation.

\section{Appendix E: Energy-Momentum Fluctuations}

Just as there are uncertainties in spacetime measurements, there are also uncertainties in energymomentum measurements due to spacetime foam effects. Thus there is a limit to how accurately we can measure and know the energy and momentum of a system [2]. Imagine sending a particle of momentum $p$ to probe a certain structure of spatial extent $l$ so that $p \sim \hbar / l$. It follows that $\delta p \sim\left(\hbar / l^{2}\right) \delta l$. Spacetime fluctuations $\delta l \gtrsim l\left(l_{P} / l\right)^{2 / 3}$ can now be used to give

$$
\delta p=\beta p\left(\frac{p}{m_{P} c}\right)^{2 / 3}, \quad \delta E=\gamma E\left(\frac{E}{E_{P}}\right)^{2 / 3},
$$


where a priori $\beta \sim 1$ and $\gamma \sim 1, E_{P}=m_{P} c^{2} \sim 10^{19} \mathrm{GeV}$ is the Planck energy and we have added the corresponding statement for energy uncertainties. We emphasize that all the uncertainties take on \pm sign with equal probability (most likely, something like a Gaussian distribution about zero).

\subsection{Modified Dispersion Relations}

Energy-momentum uncertainties affect both the energy-momentum conservation laws and dispersion relations [43]. Energy-momentum is conserved up to energy-momentum uncertainties due to quantum foam effects, i.e., $\Sigma\left(p_{i}^{\mu}+\delta p_{i}^{\mu}\right)$ is conserved, with $p_{i}^{\mu}$ being the average values of the various energymomenta. On the other hand the dispersion relation is now generalized to read

$$
E^{2}-p^{2} c^{2}-\epsilon p^{2} c^{2}\left(\frac{p c}{E_{P}}\right)^{2 / 3}=m^{2} c^{4}
$$

for high energies with $E \gg m c^{2}$. A priori we expect $\epsilon \sim 1$ and is independent of $\beta$ and $\gamma$. But since the dispersion relation is actually derived from $(E+\delta E)^{2}-(p+\delta p)^{2} c^{2}=m^{2} c^{4}$, with $\delta p$ and $\delta E$ given by Eqs. (14), the coefficients in Eqs (14) and (15) are related as

$$
\epsilon=2(\beta-\gamma)
$$

\subsection{Fluctuating Speed of Light}

The modified dispersion relation discussed above has an interesting consequence for the speed of light [44]. Applying Eq. (15) to the massless photon yields its speed [43]

$$
v=\frac{\partial E}{\partial p} \simeq c\left(1+\frac{5}{6} \epsilon \frac{E^{2 / 3}}{E_{P}^{2 / 3}}\right),
$$

which is energy-dependent and fluctuates around c. For example, a photon of $10^{13} \mathrm{eV}$ energy has a speed fluctuating about $c$ by about $1 \mathrm{~cm} / \mathrm{sec}$.

\subsection{Unmodified Threshold Energies in Collisions}

Consider the scattering process in which an energetic particle of mass $m_{1}$ collides head-on with a soft photon of energy $\omega$ in the production of two energetic particles with mass $m_{2}, m_{3}$, as in the interaction of an ultra-high energy cosmic particle with the Cosmic Microwave Background Radiation. After taking into account energy-momentum uncertainties, energy-momentum conservation, and the modified dispersion relations Eq. (15), we obtain the threshold energy equation [43]

$$
E_{t h}=p_{0}+\tilde{\eta} \frac{1}{4 \omega} \frac{E_{t h}^{8 / 3}}{E_{P}^{2 / 3}},
$$

where $p_{0} \equiv\left[\left(m_{2}+m_{3}\right)^{2}-m_{1}^{2}\right] / 4 \omega$ is the (ordinary) threshold energy if there are no energy-momentum uncertainties, and $\tilde{\eta} \equiv \eta_{1}-\left[\eta_{2} m_{2}^{5 / 3}+\eta_{3} m_{3}^{5 / 3}\right] /\left(m_{2}+m_{3}\right)^{5 / 3}$, with $\eta_{i} \equiv 2 \beta_{i}-2 \gamma_{i}-\epsilon_{i}$. With the aid of Eq. (16), we obtain $\eta_{i}=0$. It follows that the threshold energies are not modified. Now recall that, a priori, quantum fluctuations can lower as well as raise the reaction thresholds. But the lowering of reaction thresholds can give rise to matter instability which is not observed, as pointed out by Aloisio et al. [45]. With the threshold energies not modified, the serious problem of matter instability is avoided. 


\section{Appendix F: Gamma Ray \& Cosmic Ray Phenomenologies}

\subsection{High Energy $\gamma$ Rays from Distant GRB}

Recall that, due to quantum fluctuations of spacetime, the speed of light fluctuates around $c$ and the fluctuations increase with energy $\left(\delta v \sim c\left(E / E_{P}\right)^{2 / 3}\right.$, according to Eq. (17)). Thus for photons emitted simultaneously from a distant source, we expect an energy-dependent spread in their arrival times. To maximize the spread in arrival times, we should look for energetic photons from distant sources. So the idea is to look for a noticeable spread in arrival times for high energy gamma rays from distant gamma ray bursts. This proposal was first made by G. Amelino-Camelia et al. [44] in another context. But the time-of-flight differences $\delta t$ increase only with the cube root of the average overall time $t$ of travel ( $\delta t \sim t^{1 / 3} t_{P}^{2 / 3}$ ) from the gamma ray bursts to our detector, leading to a time spread too small to be detectable [43]. Thus, if the spread in arrival times for the photons recently observed [46] by the MAGIC gamma-ray telescope during a flare of the active galaxy Markarian 501 is indeed confirmed to be due to quantum gravity effects [47], then these effects are beyond those associated with quantum foam discussed in this article.

\subsection{Ultra-High Energy Cosmic Ray Events}

Theoretically one expects the UHECRs to interact with the Cosmic Microwave Background Radiation and produce pions. These interactions above the threshold energy should make observations of UHECRs with $E>6 \cdot 10^{19} \mathrm{eV}$ (the GZK limit) unlikely. Since the reaction threshold energies are not modified, we expect no violation of the GZK bound due to quantum foam effects. (However this bound does not apply to cosmic rays coming from nearby sources [48].)

\section{References and Notes}

1. (a) Wheeler, J.A. In Relativity, Groups and Topology; DeWitt, B.S.; DeWitt, C.M., Ed.; Gordon \& Breach: New York, 1963; p 315. (b) Hawking, S.W.; Page, D.N.; Pope, C.N. Quantum Gravitational Bubbles. Nucl. Phys. 1980, 170, 283-306. (c) Ashtekar, A.; Rovelli, C.; Smolin, L. Weaving a Classical Geometry with Quantum Threads. Phys. Rev. Lett. 1992, 69, 237-240. (d) Ellis, J.; Mavromatos, N.; Nanopoulos, D.V. String Theory Modifies Quantum Mechanics. Phys. Lett. 1992, B 293, 37-48. (e) Garay, L.J. Quantum Gravity and Minimum Length. Int. J. Mod. Phys. 1995, A 10, 145-166. (f) Diosi, L.; Lukacs, B. On the Minimum Uncertainty of Space-Time Geodesics. Phys. Lett. 1989, A 142, 331-334. (g) Kempf, A.; Mangano, G.; Mann, R.B. Hilbert Space Representation of the Minimum Length Uncertainty Relation. Phys. Rev. 1995, D 52, 1108-1118. (h) Hossenfelder, S. The Miminal Length and Large Extra-Dimensions. Mod. Phys. Lett. 2004, A 19, 2727-2744.

2. (a) Ng, Y.J.; van Dam, H. Limit to Spacetime Measurement. Mod. Phys. Lett. 1994, A 9, 335340. (b) Ng, Y.J.; van Dam, H. Remarks on Gravitational Sources. Mod. Phys. Lett. 1995, A 10, 2801-2808.

3. (a) Karolyhazy, F. Gravitation and Quantum Mechanics of Macroscopic Objects. Il Nuovo Cimento 
1966, A 42, 390-402. (b) Sasakura, N. An Uncertainty Relation of Space-Time. Prog. Theor. Phys. 1999, 102, 169-179.

4. Ng, Y.J.; van Dam, H. Measuring the Foaminess of Spacetime with Gravity-Wave Interferometers. Found. Phys. 2000, 30, 795-805.

5. Ng, Y.J. From Computation to Black Holes and Space-time Foam. Phys. Rev. Lett. 2001, 86, 2946-2949; (erratum) 2002, 88, 139902.

6. Lloyd, S.; Ng, Y.J. Black Hole Computers. Sci. Am. 2005, 291, \#5, 52-61.

7. (a) Christiansen, W.A.; Ng, Y.J.; van Dam, H. Probing Spacetime Foam with Extragalactic Sources. Phys. Rev. Lett. 2006, 96, 051301-1 - 051301-4; (erratum) 2007, 98, 259903. (b) Christiansen, W.A.; Ng, Y.J.; van Dam, H. Reply to Diosi's Comment on "Probing Spacetime Foam with Extragalactic Sources”. Phys. Rev. Lett. 2007, 98, 259002.

8. (a) Arzano, M.; Kephart, T.W.; Ng, Y.J. From Spacetime Foam to Holographic Foam Cosmology. Phys. Lett. 2007, B 649, 243-246. (b) Maziashvili, M. Space-Time in Light of Karolyhazy Uncertainty Relation. Int. J. Mod. Phys. 2007, D 16, 1531-1539.

9. Ng, Y.J. Holographic Foam, Dark Energy and Infinite Statistics. Phys. Lett. 2007, B 657, 10-14.

10. Requardt, M. About the Minimal Resolution of Space-Time Grains in Experimental Quantum Gravity. arXiv:0807.3619[gr-qc].

11. Giovannetti, V.; Lloyd, S.; Maccone, L. Quantum-Enhanced Measurements: Beating the Standard Quantum Limit. Science 2004, 306, 1330-1336.

12. Margolus, N.; Levitin, L.B. The Maximum Speed of Dynamical Evolution. Physica 1998, D 120, 188-195.

13. Note the qualification "average". This result is not inconsistent with that found in Calmet, X.; Graesser, M.; Hsu, S. D. Minimum Length from Quantum Mechanics and Classical General Relativity. Phys. Rev. Lett. 2004, 93, 211101-1 - 211101-4.

14. (a) 't Hooft, G. In Salamfestschrift; Ali, A. et al., Ed.; World Scientific: Singapore, 1993; p 284. (b) Susskind, L. The World as a Hologram. J. Math. Phys. (N.Y.) 1995, 36, 6377-6396. (c) Bekenstein, J.D. Black Holes and Entropy. Phys. Rev. 1973, D 7, 2333-2346. (d) Hawking, S. Particle Creation by Black Holes. Comm. Math. Phys. 1975, 43, 199-220. (e) Giddings, S.B. Black Holes and Massive Remnants. Phys. Rev. 1992, D 46, 1347-1352. (f) Bousso, R. The Holographic Principle. Rev. Mod. Phys. 2002, 74, 825-874.

15. (a) Gambini, R.; Pullin, J. Holography in Spherically Symmetric Loop Quantum Gravity. arXiv:0708.0250 [gr-qc]. (b) Husain, V. (unpublished).

16. (a) Amelino-Camelia, G. Limits on the Measurability of Space-Time Distances in the Semi-Classical Approximation of Quantum Gravity. Mod. Phys. Lett. 1994, A 9, 3415-3422. (b) Amelino-Camlia, G. An Interferometric Gravitational Wave Detector as a Quantum-Gravity Apparatus. Nature 1999, 398, 216-218.

17. For related effects of quantum fluctuations of spacetime geometry, see (a) Ford, L.H. Gravitons and Light Cone Fluctuations. Phys. Rev. 1995, D 51, 1692-1700. (b) Thompson, R. H.; Ford, L. H. Spectral line broadening and angular blurring due to spacetime geometry fluctuations. Phys. Rev. 2006, D 74, 024012-1 - 024012-12. (c) Hu, B. L.; Shiokawa, K. Wave propagation in stochastic spacetime: Localization, amplification, and particle creation. Phys. Rev. 1998, D 57, 3474-3483. 
(d) Hu, B.L.; Vergaguer, E. Stochastic Gravity: Theory and Applications. Living Rev. Rel. 2004, 7, 3.

18. Ng, Y.J.; Christiansen, W.A.; van Dam H. Probing Planck-scale Physics with Extragalacic Sources? Astrophys. J. 2003, 591, L87-L90.

19. Lieu, R.; Hillman, L.W. The Phase Coherenece of Light from Extragalactic Sources - Direct Evidence Against First Order Planck Scale Fluctuations in Time and Space. Astrophys. J. 2003, 585, L77-L80.

20. Perlman E.S. et al. The Apparent Host Galaxy of PKS 1413+135: HST, ASCA and VLBA Observations. Astro. J. 2002, 124, 2401-2412.

21. Lloyd, S. Computational Capacity of the Universe. Phys. Rev. Lett. 2002, 88, 237901-1 - 237901-4.

22. Krauss, L.M.; Turner, M.S. Geometry and Destiny. Gen. Rel. Grav. 1999, 31, 1453-1459.

23. (a) Perlmutter, S. et al. Measurements of Omega and Lambda from 42 High-Redshift Supernovae. Astrophys. J. 1999, 517, 565-586. (b) Riess, A.G. et al. Observational Evidence from Supernovae for an Accelerating Universe and a Cosmological Constant. Astron. J. 1998, 116, 1009-1038. (c) Bennett, C.L. et al. First Year Wilkenson Microwave Anisotropy Probe (WMAP) Observations: Preliminary Maps and Basic Results. Astrophys. J. Suppl. 2003, 148, 1. (d) Allen, S.W. et al. Cosmological Constraints from the Local X-Ray Luminosity Function of the Most X-Ray Luminous Galaxy Clusters. Mon. Not. Roy. Astron. Soc. 2003, 342, 287-29.

24. (a) Doplicher, S.; Haag, R.; Roberts, J. Local Observables and Particle Statistics I. Commun. Math. Phys. 1971, 23, 199-230. (b) Doplicher, S.; Haag, R.; Roberts, J. Local Observables and Particle Statistics II. Commun. Math. Phys. 1974, 35, 49-85.

25. Govorkov, A.B. Parastatistics and Parafields. Theor. Math. Phys. 1983, 54, 234-241.

26. Greenberg, O.W. Example of Infinite Statistics. Phys. Rev. Lett. 1990, 64, 705-708.

27. Fredenhagen, K. On the Existence of Antiparticles. Commun. Math. Phys. 1981, 79, 141-151.

28. For work on nonlocality in theories using Hopf algebra or Moyal-plane, see (a) Arzano, M. Quantum Fields, Non-Locality and Quantum Group Symmetries. arXiv:0710.1083 [hep-th]. (b) Balachandran, A.P. et al. S-Matrix on the Moyal Plane: Locality versus Lorentz Invariance. arXiv:0708.1379 [hep-th].

29. (a) Jejjala, V.; Kavic, M.; Minic, D. Fine Structure of Dark Energy and New Physics. arXiv:0705.4581 [hep-th]. (b) Benatti, F.; Floreanini, R. Non-Standard Neutral Kaons Dynamics from D-Brane Statistics. Annals Phys. 1999, 273, 58-71. (c) Medved, A. J. M. A comment or two on holographic dark energy. arXiv:0802.1753 [hep-th].

30. (a) Strominger, A. Black Hole Statistics. Phys. Rev. Lett. 1993, 71, 3397-3400. (b) Volovich, I.V. D-branes, Black Holes and $S U(\infty)$ Gauge Theory. arXiv:hep-th/9608137 and references therein.

31. Giddings, S.B. Black Holes, Information, and Locality. arXiv:0705.2197 [hep-th].

32. Horowitz, G.T. Black Holes, Entropies, and Information. arXiv:0708.3680 [astro-ph].

33. Ahluwalia, D.V. Quantum Measurements, Gravitation, and Locality. Phys. Lett. 1994, B 339, 301-303.

34. (a) Chen, W.; Wu, Y.S. Implications of a Cosmological Constant Varying as $R^{-2}$. Phys. Rev. 1990, D 41, 695-698. (b) Sorkin, R.D. Forks in the Road, on the Way to Quantum Gravity. Int. J. Theor. Phys 1997, 36, 2759-2781. (c) Ng, Y.J.; van Dam, H. A Small but Nonzero Cosmological Constant. 
Int. J. Mod. Phys. 2001, D 10, 49-55.

35. van der Bij, J.J.; van Dam, H.; Ng, Y.J. The Exchange of Massless Spin-Two Particles. Physica 1982, A116, 307-320.

36. (a) Zimdahl, W.; Pavon, D. Interacting Holographic Dark Energy. arXiv:0606555 [astro-ph]. (b) Biswas, T. et al. Current Acceleration from Dilaton and Stringy Cold Dark Matter. Phys. Rev. 2006, D 74, 063501-1 - 063501-13. (c) Zimdahl, W. Dark Energy: A Unifying View. arXiv:0705.2131 [gr-qc]. (d) Abdalla, E. et al. Signature of the interaction between dark energy and dark matter in galaxy clusters. arXiv:0710.1198 [astro-ph]. (e) Pavon, D.; Wang, B. Le Chatelier-Braun principle in cosmological physics. arXiv:0712.0565 [gr-qc].

37. For more discussions on holographic cosmology, see, e.g., (a) Fischler, W.; Susskind, L. Holography and Cosmology. arXiv:hep-th/9806039. (b) Easther, R.; Lowe, D. Holography, Cosmology, and the Second Law of Thermodynamics. Phys. Rev. Lett. 1999, 82, 4967-4970. (c) Veneziano, G. Prebangian Origin of our Entropy and Time Arrow. Phys. Lett. 1999, B 454, 22-26. (d) Kaloper, N.; Linde, A. Cosmology versus Holography. Phys. Rev. 1999, D 60, 103509-1 - 103509-7. (e) Cohen, A.G. et. al. Effective Field Theory, Black Holes, and the Cosmological Constant. Phys. Rev. Lett. 1999, 82, 4971-4974. (f) Horava, P.; Minic, D. Probable Values of the Cosmological Constant in a Holographic Theory. Phys. Rev. Lett. 2000, 85, 1610-1613. (g) Thomas, S. Holography Stabilizes the Vacuum Energy. Phys. Rev. Lett. 2002, 89, 081301-1 - 081301-4 . (h) Hsu, S.D.H. Entropy Bounds and Dark Energy. Phys. Lett. 2004, B 594, 13-16. (i) Li, M. A Model of Dark Energy. Phys. Lett. 2004, B 603, 1-5. (j) Lee, J.-W.; Lee, J.; Kim, H.-C. Dark energy from vacuum entanglement. arXiv:hep-th/0701199. (k) Cai, R.-G. A dark energy model characterized by the age of the Universe. arXiv:0707.4049 [hep-th]. (1) Horvat, R. Holographic dark energy: quantum correlations against thermodynamical description. arXiv:0711.4013 [gr-qc]. (m) Padmanabhan, T. Emergent Gravity and Dark Energy. arXiv:0802.1798 [gr-qc]. (n) Ma, Y. Z.; Gong, Y.; Chen, X. Features of holographic dark energy under the combined cosmological constraints. arXiv:0711.1641 [astro-ph].

38. For a related view on holography, information theory and inflationary universe, see (a) Zizzi, P.A. Quantum Foam and de Sitter-like Universe. Int. J. Theor. Phys. 1999, 38, 2333-2348. (b) Zizzi, P.A. Holography, Quantum Geometry, and Quantum Information Theory. Entropy 2000, 2, 39-69.

39. Salecker, H.; Wigner, E.P. Quantum Limitations of the Measurement of Space-Time Distances. Phys. Rev. 1958, 109, 571-577.

40. Barrow, J.D. Wigner Inequalities for a Black Hole. Phys. Rev. 1996, D54, 6563-6564.

41. Gambini, R.; Porto, R; Pullin, J. Fundamental Decoherence from Quantum Gravity: A Pedagogical Review. Gen. Rel. Grav. 2007, 39, 1143-1156.

42. Some of the viewpoints on this matter can be found in, e.g., (a) Susskind, L.; Lindesay, J. An Introduction to Black Holes, Information and the String Theory Revolution: The Holographic Universe; World Scientific Publishing: Singapore, 2005. (b) Ashtekar, A.; Baez, J. C.; Corichi, A.; Krasnov, K. Quantum geometry and black hole entropy. Phys. Rev. Lett. 1998, 80, 904-907. (c) Frampton, P. H.; Hsu, S. D. H.; Reeb, D.; Kephart, T. W. What is the entropy of the Universe? arXiv:0801.1847 [hep-th].

43. Ng, Y.J. In Proceedings of the Fortieth Karpacz Winter School on Theoretical Physics; KowalskiGlikman, J; Amelino-Camelia, G., Ed.; Springer: Berlin, 2005; p 321. 
44. (a) Amelino-Camelia, G.; Ellis, J.; Mavromatos, N.E.; Nanopoulos, D.V. Distance Measurement and Wave Dispersion in a Liouville String Approach to Quantum Gravity. Int. J. Mod. Phys. 1997, A12, 607-623. (b) Amelino-Camelia, G.; Ellis, J.; Mavromatos, N.E.; Nanopoulos, D.V.; Sarkar, S. Tests of Quantum Gravity from Observations of $\gamma$-Ray Bursts. Nature 1998, 393, 763-765.

45. (a) Aloisio, R. et al. A Fluctuating Energy-Momentum May Produce an Unstable World. Astropart. Phys. 2003, 20, 369-376. (b) Le Gallou, R. New Constraints on Space-Time Planck Scale Fluctuations from Established High Energy Astronomy Observations. Astropart. Phys. 2004, 20, 703-708.

46. Albert, J. et al. [MAGIC collaboration] Variable VHE Gamma-Ray Emission from Markarian 501. Astrophys. J. 2007, 669, 862-883.

47. Albert, J. et al. Probing Quantum Gravity Using Photons from a Mkn 501 Flare Observed by MAGIC. arXiv:0708.2889 [astro-ph].

48. The Pierre Auger collaboration. Correlation of the Highest-Energy Cosmic Rays with Nearby Extragalactic Objects. Science 2007, 318, 938-943.

(c) 2008 by the authors; licensee Molecular Diversity Preservation International, Basel, Switzerland. This article is an open-access article distributed under the terms and conditions of the Creative Commons Attribution license (http://creativecommons.org/licenses/by/3.0/). 